\title{
A evolução dos manuais de instrução das próteses auditivas sob a perspectiva do design
}

The evolution of hearing aid instruction manuals under the design perspective

\author{
Camila Medina, Deborah Viviane Ferrari, Cassia Leticia Carrara Domiciano
}

\begin{abstract}
Design gráfico, manuais de instrução, deficiência auditiva, aparelho auditivo
Materiais gráficos instrucionais voltados aos usuários de próteses auditivas evoluiram, revelando caraterísticas gráficas, técnicas e o papel dos impressos no processo de informação, socialização e inclusão destes usuários ao longo da história. Estes materiais foram levantados e analisados, visando contribuir para uma investigação mais ampla em andamento, que se foca no Design da Informação, no Design Instrucional e no Design Gráfico Inclusivo voltado a um público muitas vezes negligenciado. A análise constatou que o contexto histórico e a evolução dos dispositivos, aliados aos movimentos artísticos e processos gráficos vigentes influenciaram diretamente na apresentação gráfica e textual dos manuais de instrução.
\end{abstract}

\section{Graphic Design, instruction materials, hearing loss, hearing aid}

Instructional graphic materials for hearing aids users evolved revealing graphic features, techniques and printed material roles for the information, socialization and inclusion of such users along history. These materials were gathered and analyzed in order to contribute to a broader ongoing research, which focuses on Information Design, Instructional Design and Inclusive Design aimed at an often overlooked public. The analysis revealed that historical context and devices evolution, together with artistic movements and graphic processes have influenced directly the graphic presentation of the instruction materials.

\section{Introdução}

A perda auditiva permanente impacta negativamente a comunicação oral, o bem estar psicossocial e qualidade de vida do indivíduo. Instrumentos para reduzir tais consequências datam de vários séculos, sendo progressivo o aumento de sua eficiência. De modo geral, a tecnologia destes dispositivos é dividida em Era Acústica e Era Eletrônica, sendo que esta última se subdivide em carbono, válvula, transistor, circuito integrado, microprocessadores e era digital (MUDRY; DODELÉ, 2000) conforme a Figura 1.

Quando as próteses auditivas começaram a ser comercializadas, materiais instrucionais passaram a acompanhar os produtos: são os chamados manuais de instrução. Em formato de folheto ou livro, associam texto a conteúdo gráfico e atualmente, encontram-se também em formato digital. É fundamental ao sucesso do tratamento que o usuário leia e retenha informações sobre a utilização e manutenção adequada da prótese (BROOKE et al, 2012). 
Figura 1: A linha do tempo do Aparelho de Amplificação Sonora Individual (AASI) - Adaptação (FERRARI, 2014 material didático, com permissão da autora)

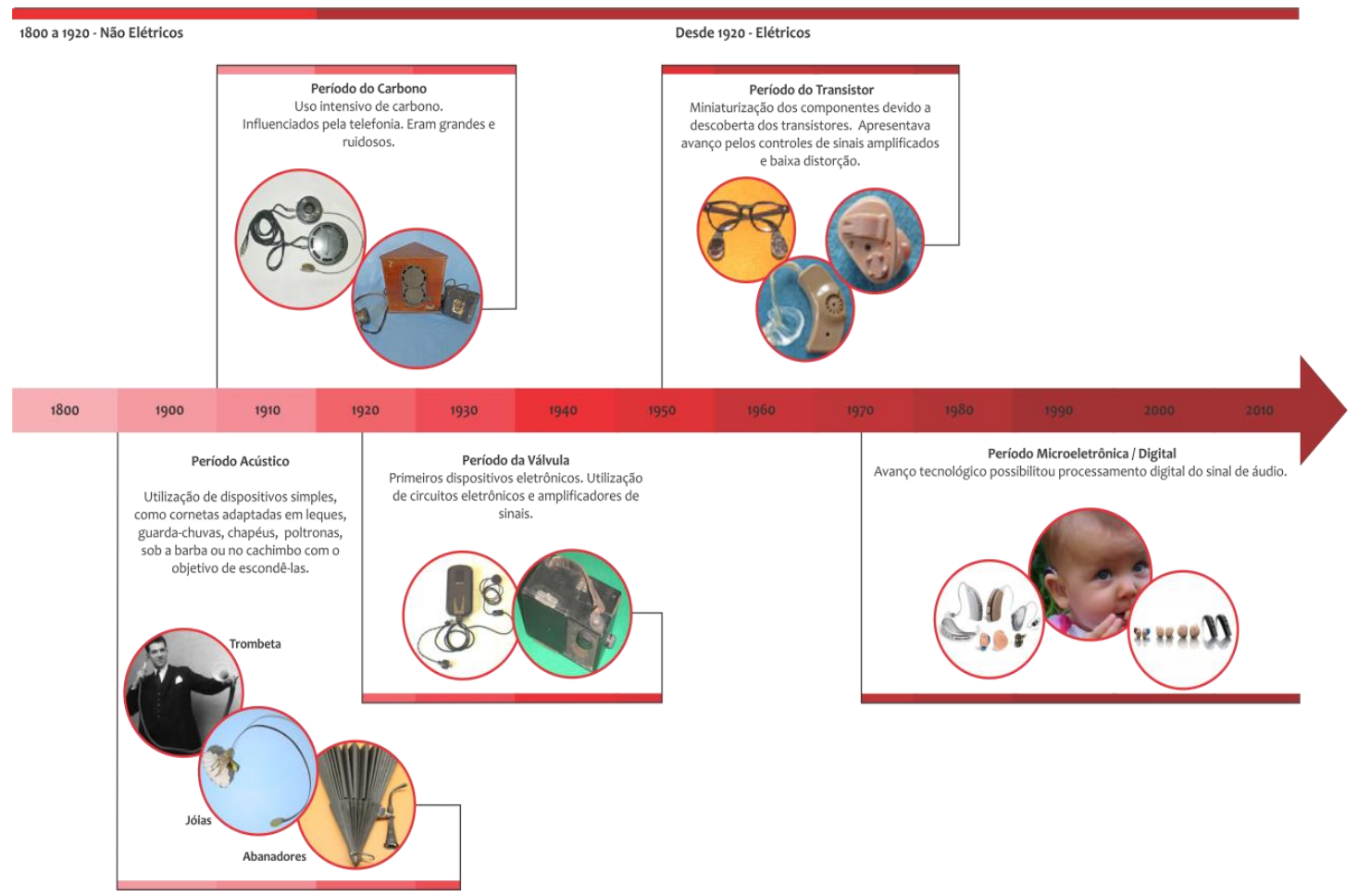

Esses manuais adaptaram-se ao contexto de cada época, influenciados pela evolução da tecnologia dos aparelhos auditivos, pelo incremento dos processos gráficos e pela história das artes e design. O Design é uma área que pode propor intervenções projetuais que facilitem a comunicação com estes pacientes.

O objetivo deste estudo é levantar e analisar os manuais de instrução de próteses auditivas, inter-relacionando-os com a evolução tecnológica, o contexto histórico do design e com as técnicas de produção gráfica.

\section{Questões metodológicas}

Foram encontrados diversos manuais de instrução de próteses auditivas de diferentes épocas, e cinco destes foram selecionados para análise. A busca foi feita no acervo digital do Museu do Aparelho Auditivo e em sites especializados. O levantamento aborda desde o primeiro registro encontrado, 1890 , até os dias atuais. Tal análise foi feita à luz de uma bibliografia voltada às metodologias em projetos de design gráfico - Ambrose \& Harris (2012), Samara (2010, 2011), e Lupton (2014) - e à produção gráfica (Bann, 2010; Vilas Boas, 2009). Focou-se os tópicos listados na tabela 1, pois facilitam a apreciação das peças e criam um padrão comparativo. Cada manual foi analisado e gerou uma tabela completa, usada depois para discussões e análises posteriores. Como alguns manuais só foram encontrados em formato digital (sobretudo os mais antigos), nem sempre foi possível a análise acerca do formato, tipo de papel, quantidade de páginas e forma de encadernação. 
Tabela 1 - Seis tópicos e seus respectivos itens de análise dos impressos (adaptado de AMBROSE e HARRIS, 2012)

\begin{tabular}{l|l} 
Formato & Tamanho e proporção \\
\hline Layout e grids & Grid \\
& Ritmo \\
& Hierarquia de informação \\
\hline Tipografia & Anatomia e personalidade da fonte \\
& Sistemas de classificação \\
& Família \\
& Espacejamentos e Alinhamentos \\
\hline Imagem & Classificação \\
& Técnicas de Manipulação \\
\hline Cor & Classificação \\
& Cor para impressão \\
\hline Produção Gráfica & Tipos de papel \\
& Engenharia do papel \\
& Processo de impressão \\
& Processos de acabamento
\end{tabular}

\section{Design gráfico e aparelhos auditivo: histórias e análises}

A evolução dos manuais será discutida de acordo com as diferentes eras tecnológicas.

\subsection{Aparelhos Auditivos Não Elétricos - Contextualização histórica e técnica}

O final do século 19 trouxe crescente industrialização e consolidação do capitalismo. Nascia uma nova sociedade através da ascensão da burguesia industrial e organização do proletariado. Tais fatos repercutiram nas artes através de movimentos como o Impressionismo e o Art Noveau. Nas chamadas artes gráficas, onde se incluíam as peças reproduzidas em série através de processos de impressão, destacou-se o Estilo Vitoriano. Com o Art Noveau, este estilo prezava por formas e tipografias rebuscadas ligadas à publicidade e ao consumo. $\mathrm{O}$ primeiro processo de impressão de texto mecanizado foi a Tipografia, que surgiu no século XV e perdurou até meados do XX. Os textos de produtos impressos em tipografia no século XIX eram feitos em tipos de metal e as imagens em clichê de alto relevo.

A evolução dos processos industriais proporcionou o incremento do maquinário para impressão tipográfica e produção de papel, o que tornou viável o aumento da quantidade de produtos editoriais. A prensa à vapor e a invenção da máquina mecânica de composição (Linotype) propiciou evolução e agilidade no processo. Além da tipografia, a litografia, voltada principalmente para reproduções a cores, desenvolveu-se, assim como o uso de fotografias. Nascia aí, segundo Cardoso (2005), a indústria gráfica. Esta transformação resultou em uma imensa mudança na maneira como a informação visual era produzida e difundida. A partir de 1850, o aperfeiçoamento no processo fotográfico, com o uso de negativos em substrato transparente (filmes) permitiu o desenvolvimento da fotogravura e a fotolitografia, facilitando a gravação de matrizes. Destaca-se também a invenção da Flexografia, que se utiliza, até hoje, de matrizes de borracha em alto relevo (DOMICIANO, 2012).

\subsubsection{Manuais de instrução}

A mão em concha atrás da orelha foi, possivelmente, a primeira estratégia a ser utilizada para aumento da audição. Nos séculos 18 e 19 surgiram diferentes instrumentos, como cornetas acústicas, instrumentos inseridos na orelha, instrumentos portáteis e tubos acústicos (MUDRY; DODELÉ, 2000), mas não beneficiavam indivíduos com perdas auditivas severas.

O primeiro manual encontrado é do aparelho de Condução Óssea Audiophone Bernard (1890). (MUSEU DO APARELHO AUDITIVO) 
Figuras 2 e 3: Manual de instrução e o aparelho Audiphone Bernard Fonte: Acervo digital do Museu do Aparelho Auditivo ${ }^{1}$ ( uso com permissão) disponível em <http://museudoaparelhoauditivo.com.br/acervo-aparelhos-auditivos-nao-eletricosaparelho-auditivo-de-conducao-ossea-aparelho-auditivo-de-conducao-ossea-audiphone-bernard.php>
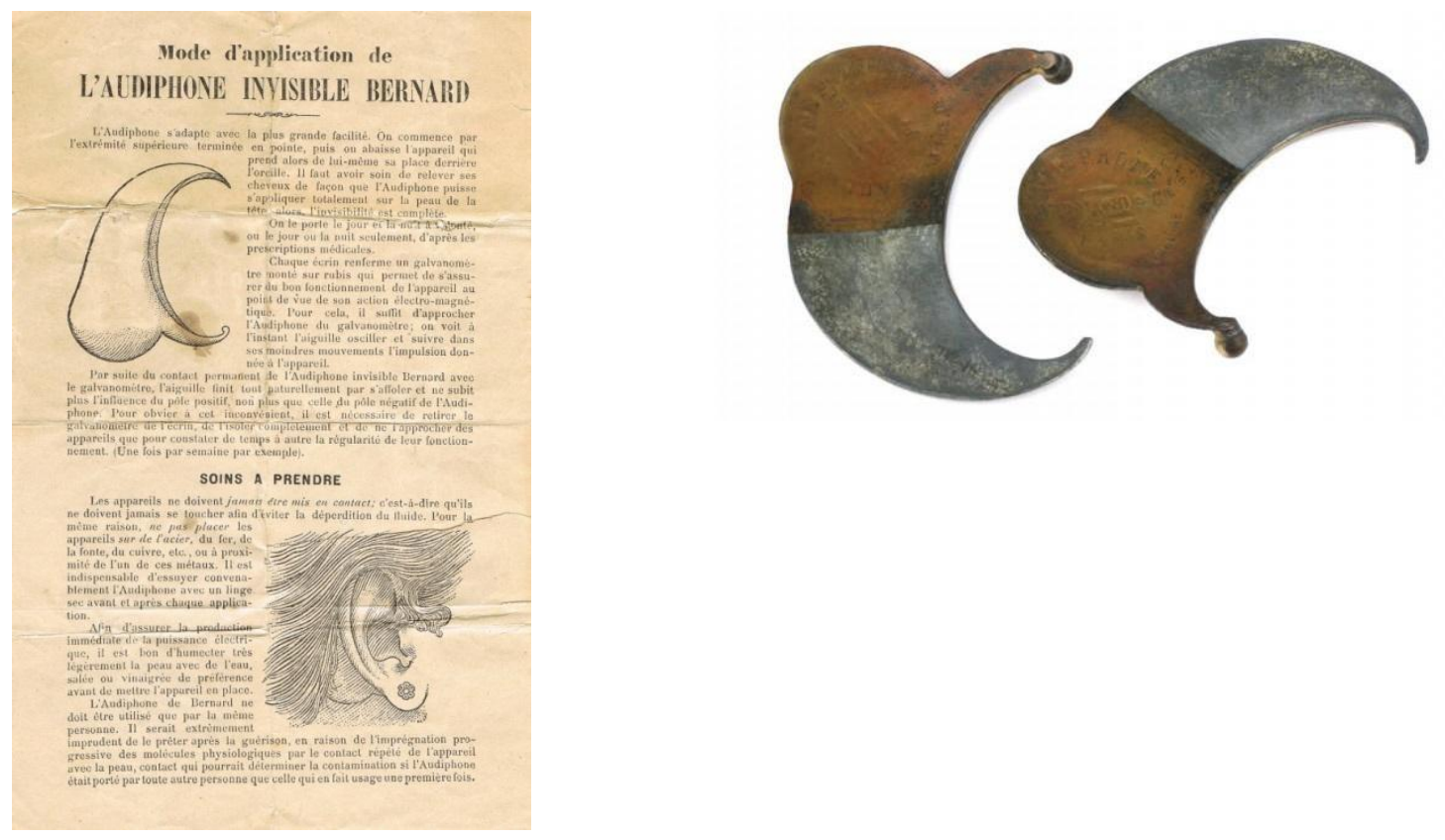

Pela data e características gráficas pode-se afirmar que a impressão foi feita em Tipografia (cor única, impressão com falhas devido à pressão), com llustrações feitas à traço, para possibilitar o uso de um clichê metálico ainda sem retículas (segundo caracterização de Bann, 2010). Pela tabela de análise (tabela 1) verificou-se uma formatação limitada pelas tecnologias de impressão, com uso de três tipografias diferentes,. O rebuscamento da figura lembra características do movimento vigente na época, o Art Noveau.

\subsection{Aparelhos Auditivos Elétricos (depois de 1920) - Contextualização histórica e técnica}

De 1920 a 1960

Nas primeiras décadas do século XX o mundo assistia a várias transformações e eventos históricos, como as Guerras Mundiais e a Revolução Russa (CARDOSO, 2005). Neste contexto surgia o Modernismo (1920 a 1960), um amplo movimento cultural, que quebrava regras anteriores na literatura e nas artes. Tal movimento foi reflexo do descontentamento em relação às condições sociais, políticas e culturais vigentes até então. Novas linguagens precisavam traduzir uma nova realidade pós guerra.

O design institucionalizou-se pela criação da Bauhaus, escola alemã, que prezava pelo design de formas simples e despojadas de ornamentos. O design do objeto gráfico, chamado até então de "artes gráficas", foi influenciado também por movimentos artísticos do período, como o cubismo, futurismo e construtivismo. O lema "A forma segue a função" refletiu-se no design gráfico com "... uma abordagem assimétrica para o layout, com uma adesão escrita ao grid, ênfase no espaço em branco e na tipografia sem serifa, na ausência da decoração e embelezamento" (AMBROSE e HARRIS, 2012).

Influenciada pelas inovações tecnológicas e atendendo à demanda em um contexto histórico efervescente, a indústria gráfica desenvolveu importantes processos de impressão: a rotogravura e o offset, uma evolução da litografia, permitindo mudanças nas características gráficas dos impressos, como uso mais preciso de tons e reprodução das cores, (DOMICIANO, 2012). 
Após o fim da Segunda Guerra Mundial (1945), a tecnologia proveniente do surgimento do computador revolucionou os processos gráficos e impulsionou uma globalização inicial. No design, a difusão de padrões e regras que definiam o "bom desenho" consolidado no modernismo deu-se pelo Novo Estilo Internacional, pela Escola Suiça e a Escola de Ulm (alemã, a "nova Bauhaus").

\section{A partir de 1960}

A contracultura e movimentos como o Hippie e a PopArt, foram contra o formalismo imposto pelos "modernos", esboçando as primeiras questões referentes a uma realidade pós-moderna. Destaca-se nesse período o avanço das tecnologias de comunicação e, posteriormente, manipulação digital de texto e imagem, que possibilitou ampliar formas de apresentação gráfica. Na indústria gráfica, o uso de computadores, periféricos e softwares criaram grande revolução, principalmente na fase da pré-impressão, que passou a ser digital. Hoje, vivemos na era da arquitetura da informação, dos produtos híbridos e da arte digital. As transformações não foram somente tecnológicas, mas sociais e culturais.

\subsubsection{Manuais de instrução}

\subsubsection{Período do Carbono (1910-1940)}

A invenção do telefone por Graham Bell (1876) e dos transmissores de carbono por Blake e Hughes (1878) permitiram que estas tecnologias fossem adaptadas para as próteses auditivas (LYBARGER; LYBARGER, 2000). Tais dispositivos não beneficiavam indivíduos com perdas mais severas. Segue exemplo.

\section{Siemens Halske Wallet and Purse Hearing Aids}

Ilustrações mostram a prótese auditiva de 1920 escondido em uma bolsa. A análise gráfica destes manuais constatou que a fotografia é a principal marca da evolução em relação aos exemplares anteriores. A impressão monocromática prejudica a visualização do produto. A quantidade de páginas e encadernação não podem ser definidas, mas a presença da numeração das páginas disponíveis sugere que tenha sido feita uma encadernação simples de 12 ou 16 páginas. Há uma preocupação maior com o tratamento dos textos, com o uso de uma tipografia única e organização clara das informações. Estas se apresentam em um ritmo sutil entre as páginas, pela variação do local em que a figura aparece. A diferença de tamanho e forma de centralização entre texto e legenda das figuras ajudam no estabelecimento da hierarquia de informação.

Figuras 4 a 6 - Manual de intrução e Aparelho Siemens Halske Wallet and Purse. Fonte: <http://beckerexhibits.wustl.edu/did/20thcent>
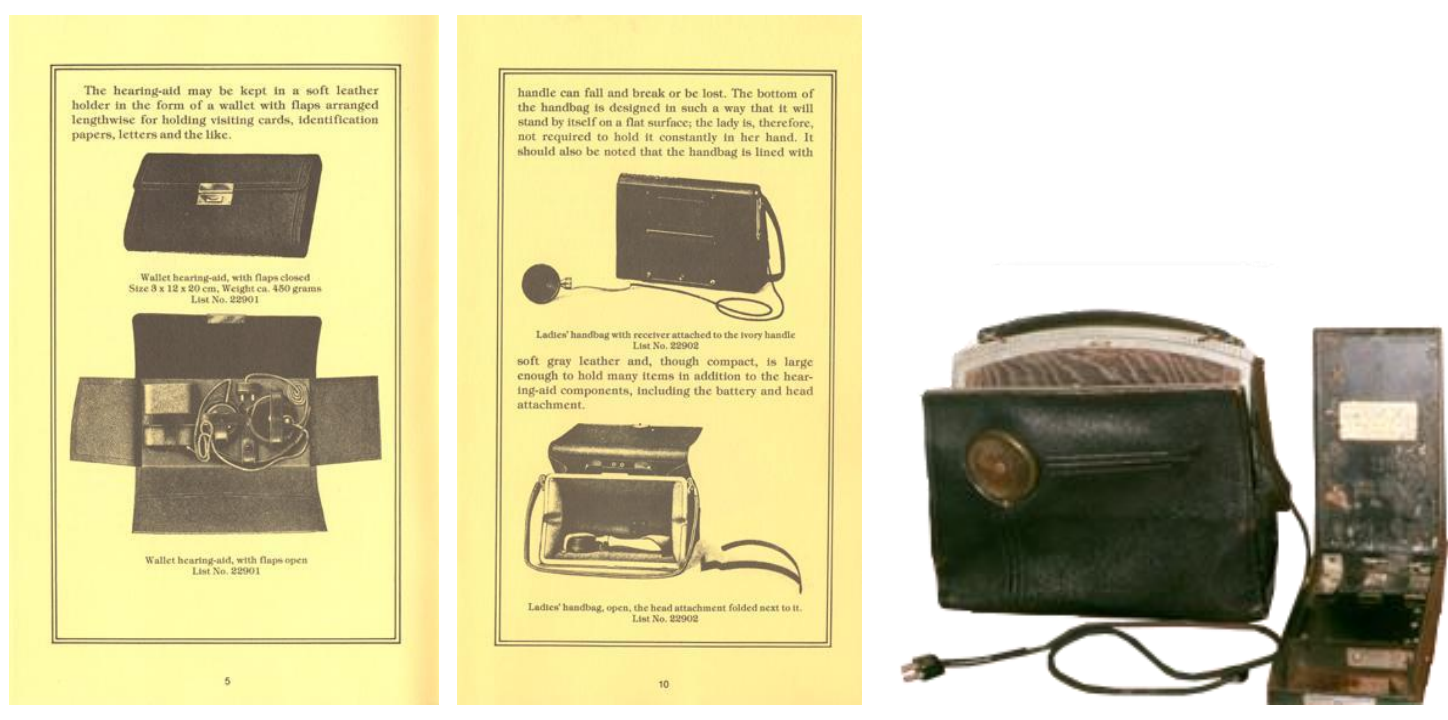


\subsubsection{Período da Válvula (1925-1955)}

A válvula de Lee De Forest (1906), abriu portas para a invensão de diferentes dispositivos. Estes forneciam maior ganho e resposta de frequência, menor distorção e maior flexibilidade de controle do que os aparelhos de carbono (LYBARGER; LYBARGER, 2000; MUDRY, DODELÉ, 2000). Segue exemplo.

\section{Radionic Hearing Aid}

Comercializado pela Zenith Radio Corporation, em 1945, era acompanhado de sua brochura. A grande inovação é a impressão nitidamente feita em offset, permitindo, entre outros ganhos, o uso de imagens fotográficas, ilustrações em tons e texturas, pela técnica do banday.

Figuras 7 a 9: Capa, contra-capa, e detalhe de duas páginas do miolo e seu respectivo aparelho Radionic. Fonte: <http://beckerexhibits.wustl.edu/did/pamphlets/Zenith/index.htm>
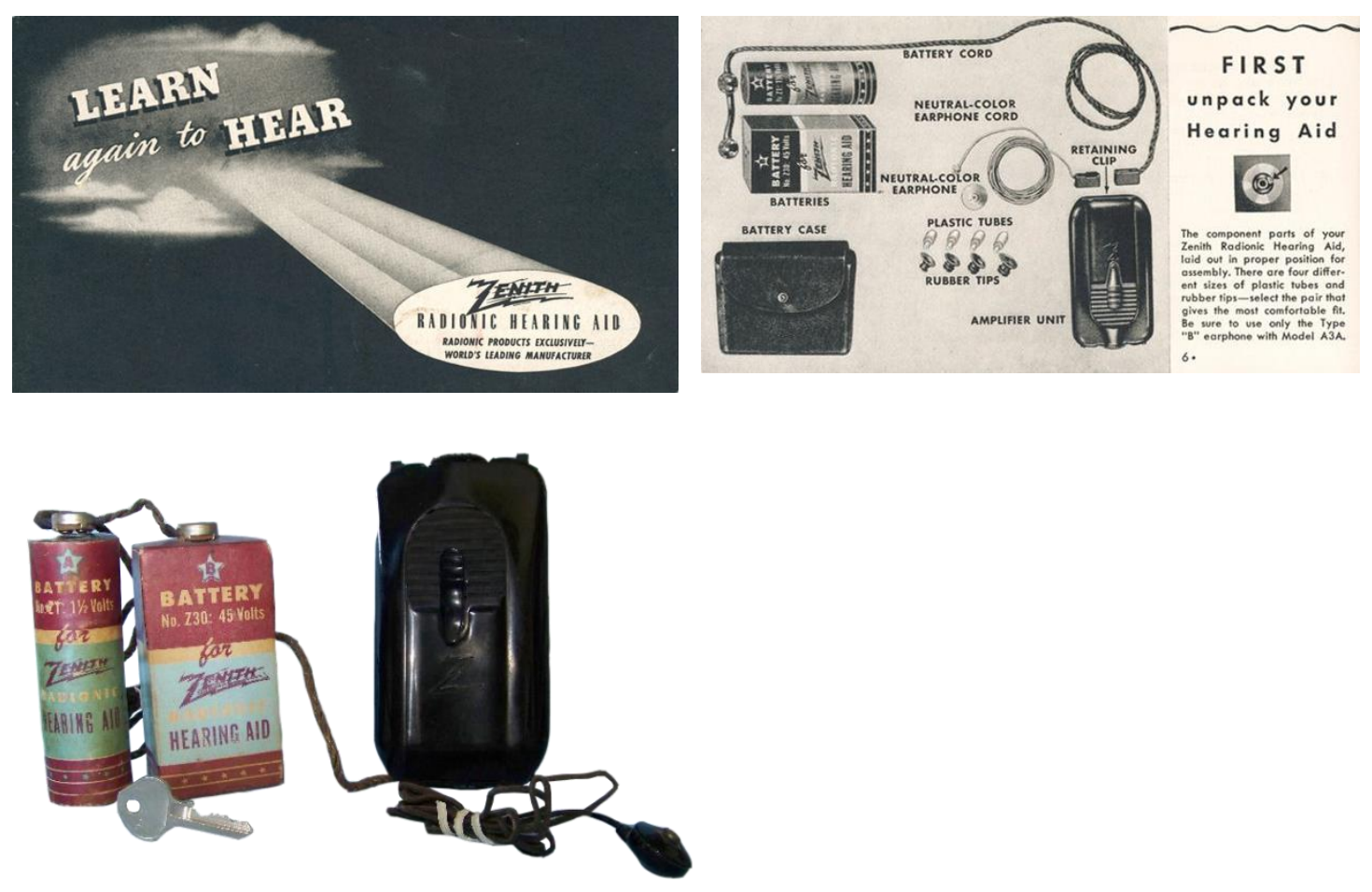

No detalhe (figuras 10 e 11), as diferentes fontes podem ser classificadas "tipo como imagem", de acordo com Ambrose e Harris (2012). Pela denominação "brochura", acredita-se que esta peça, com 44 páginas, tenha sido revestida por uma capa mole colada na lombada. Porém, não consegue-se determinar se colada, costurada ou grampeada.

Figuras 10 a 11: Detalhes do manual do dispositivo Radionic. Fonte: <http://beckerexhibits.wustl.edu/did/pamphlets/Zenith/index.htm> 

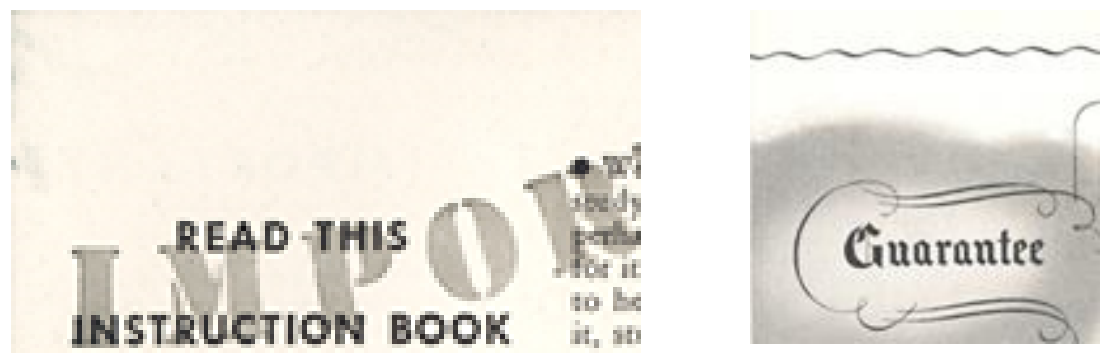

Apesar de ainda apresentar fortes características do Art Noveau, alguns traços do Modernismo aparecem, como o uso de alguns elementos decorativos, ênfase no espaço em branco e presença de tipografia sem serifa.

\subsubsection{Período do Transistor (1955-1985)}

O transistor, inventado pela Bell Telephone Laboratories (1948), trouxe melhorias dramáticas às próteses auditivas, como a miniaturização dos aparelhos e aumento da potência e resposta de freqüência dos amplificadores, alcançando as perdas mais severas (LYBARGER; LYBARGER, 2000; MUDRY, DODELÉ, 2000). Segue exemplo.

\section{Aparelho Auditivo Paravox Veri-Small, 1948.}

Primeiro impresso em mais de uma cor, este panfleto acompanhava o aparelho e mostra como escondê-lo no penteado.

Figuras 12 e 14: Capa, interior de panfleto e dispositivo Paravox. Fonte: <http://beckerexhibits.wustl.edu/did/20thcent/part4.htm>
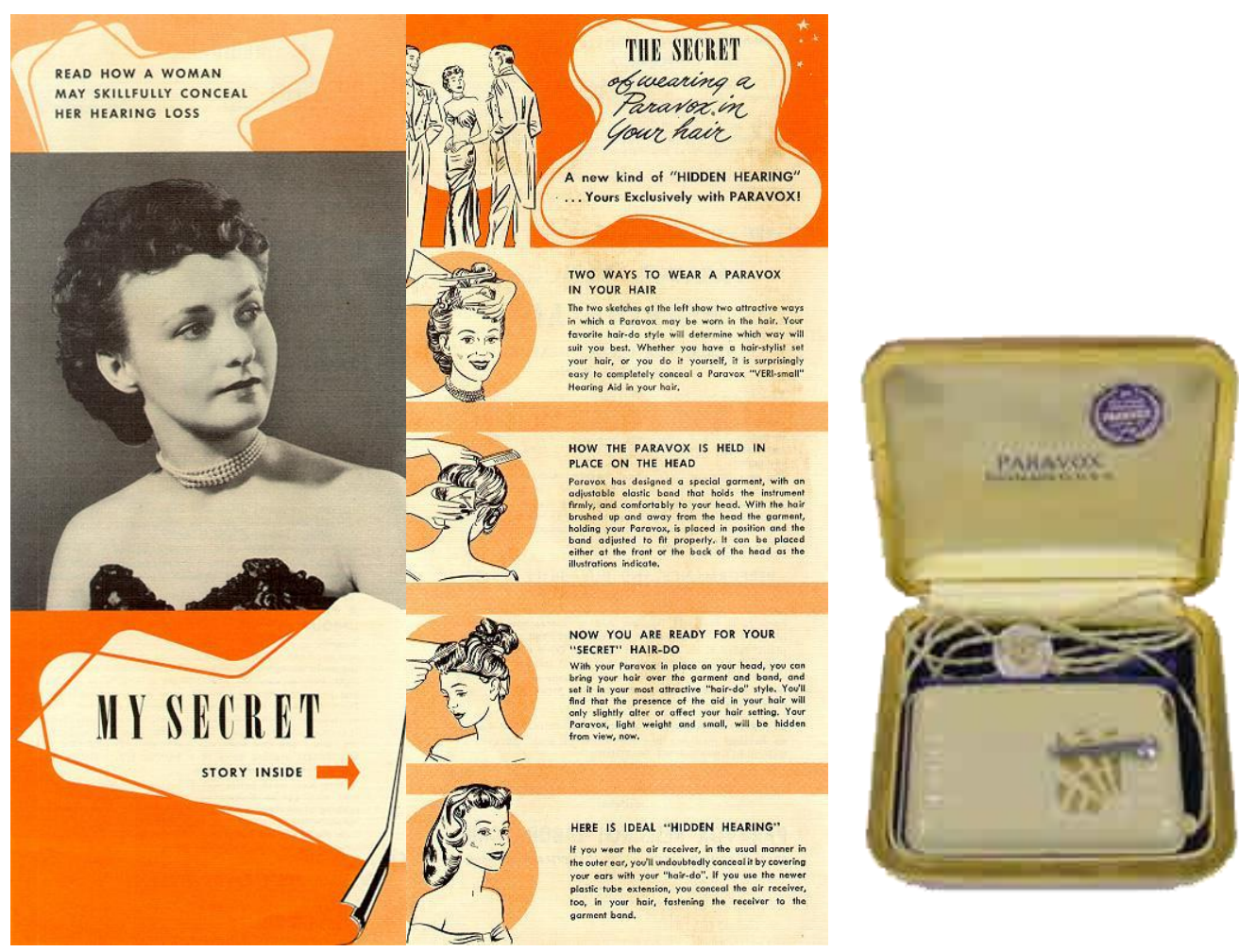

Nota-se aqui traços de simplicidade e clareza, próprios do modernismo, com uso de cores, imagens e tipografias que auxiliam no design da informação. A data da publicação e características como o uso de cores em benday propoem impressão em offset. A expressão 
"Story inside" sugere que pelo menos mais duas páginas existam neste impresso, com tamanho aproximado de $25 \times 29,4 \mathrm{~cm}$, impresso frente e verso, dobrado como um folder.

\subsubsection{Período Microeletrônica / Digital (a partir de 1970)}

A miniaturização das próteses só foi possível após o desenvolvimento do circuito integrado (1958), microfones de cerâmica (1967) e, posteriormente, de eletreto (1971). (LYBARGER; LYBARGER, 2000; MUDRY, DODELÉ, 2000).

Embora um aparelho digital de bolso tenha sido criado em 1987, somente a partir de 1996 as próteses auditiva com esta tecnologia foram empregadas e comercializadas com sucesso (MUDRY, DODELÉ, 2000). A tecnologia digital possibilita processamento e análises complexas do sinal, sendo empregada na maioria das próteses auditivas atuais. Segue exemplo.

Aparelhos auditivos Retroauriculares e microRetroauriculares Milo/MiloPlus

Este manual contempla três diferentes modelos de dispositivo. Disponíveis digitalmente em inglês e impresso em português, com mesmo layout nas duas versões.

Figuras 15 a 17: Guia do usuário Milo/MiloPlus (Fonte:

<http://www.phonak.com/content/dam/phonak/b2b/C_M_tools/Hearing_Instruments/milo/User_Guide/029_0352_02_Us er_Manual_Milo_MiloPlus_BTE.pdf>

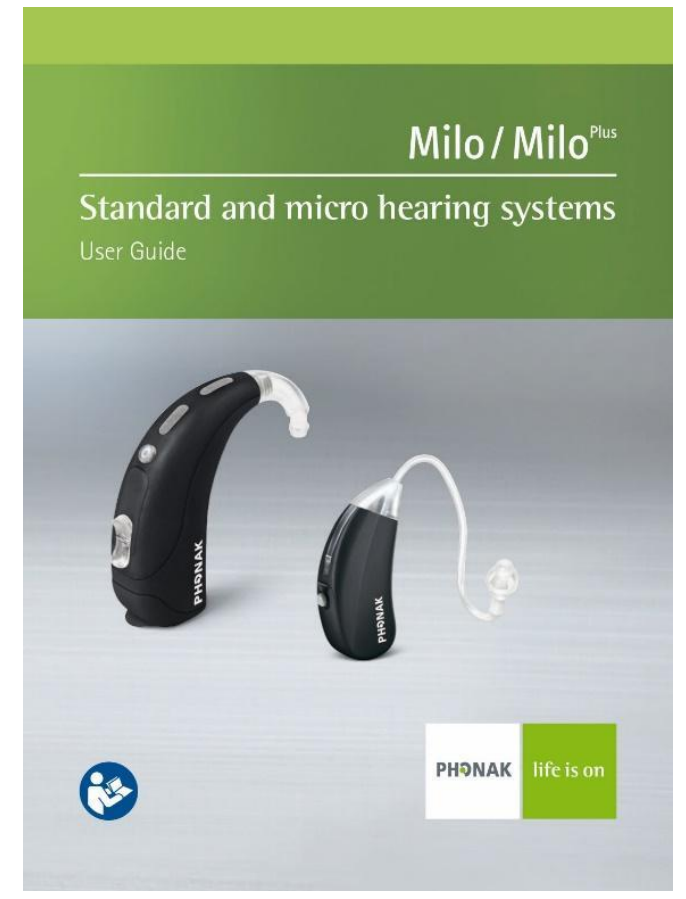

\section{Micro model}

Option B: with dome

Option C: with SlimTip
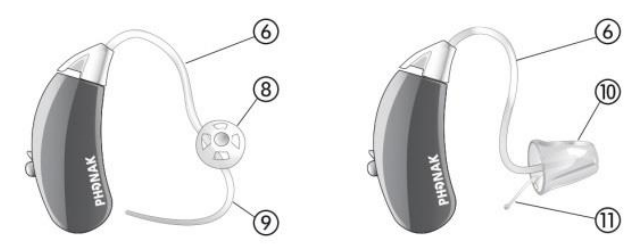
(8) Earmold: Dome
(9) Retention
(10) Earmold: SlimTip
(II) Removal handle 


\section{Troubleshooting}

Hearing systems sound softer than usual / no sound First check whether you can adjust and make the sound louder with the volume control. Then check the hearing tube and earmold for earwax residue and clean if necessary. Check whether the battery is correctly inserted and properly fitted, adjust the fit if necessary. If there is no improvement, change the battery. If the problem persists, contact your hearing care professional.

Battery discharges too quickly

Insert a new battery and make note of exactly how long it lasts. Contact your hearing care professional with this information, they will be able to help and advise you.

Crackling or buzzing noises

Check the hearing tube and earmold for earwax residue. Check the hearing tube for damage (color changes, hardening, or cracks) and contact your hearing care professional if necessary for replacement.

Impresso em offset com duas cores, no tamanho 9,5x14cm, 44 páginas. De acordo com o estudo de Capossecco et al. (2014), que avaliou 36 manuais de próteses auditivas, destacamse problemas como, a inclusão de muitos modelos de dispositivos em um só manual, uso de papel brilhante, tamanho final do impresso pequeno e tipografia menor que 12 pontos.

\section{Discussão e Considerações}

Embora o objetivo deste estudo não seja avaliar se o conteúdo técnico e instrucional estava adequado ao usuário da época, alguns elementos nos permitem delimitar características destes 125 anos de história. A análise dos manuais pelo viés da evolução dos processos gráficos e do contexto histórico possibilitou uma visão abrangente da importância de sua função social. Esta função vai além da instrução de uso e cuidados com o dispositivo e tratamento da surdez, pois um um material gráfico bem produzido pode resultar em um produto mais agradável e fácil de ler, e proporciona melhor relação do paciente com seu dispositivo, beneficiando o tratamento.

Fatores como o estigma e o isolamento social podem motivar o paciente a procurar auxílio e minimizar sua deficiência. Talvez a abordagem de pontos específicos como este seja uma resposta aos anseios do usuário e um estímulo à adesão ao uso do aparelho. Em diversas peças gráficas notou-se traços de apelo publicitário que procuravam estimular o uso e promover a inclusão do sujeito com deficiência em seu meio social. A preocupação em ocultar o dispositivo também é percebida.

Estudos atuais defendem a personalização do material, que é recomendada para que 0 paciente valorize e se simpatize com o que lhe é oferecido (BULL et al., 2001 e CAPOSECCO et al.,2014). Personalizar manuais não é uma tendência atual, pois é observada de forma rudimentar em modelo da era transistor de 1955, com folha em branco dedicada às instruções especiais, que seriam completadas de acordo com a necessidade de cada paciente.

Figura 18: Página do manual Beltone 1955 Fonte: Museu do Aparelho auditivo (uso com permissão) 


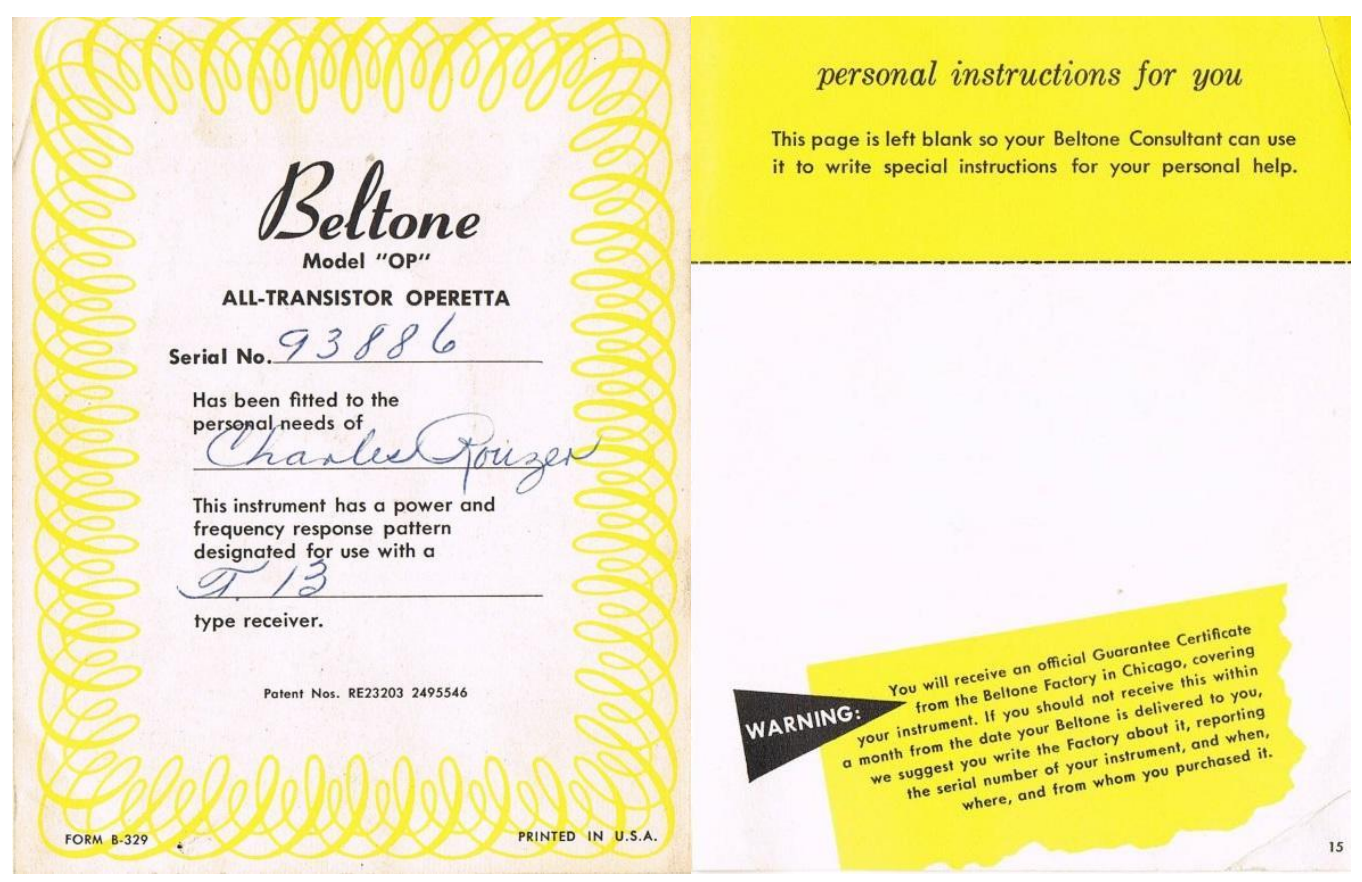

Figura 19: Página do manual Telex 1975 Fonte: Museu do Aparelho auditivo (uso com permissão)

how to keep hearing at its best...

Telex hearing glasses have been "torture tested" in heat, moisture, under severe vibration conditions, and in actual wear by users to assure you that it is rugged, dependable, and will give complete hearing satisfaction. If,
by chance, your Telex should not operate properly (perby chance, your Telex should not operate properly (per-
haps due to mishandling in transportation) check the haps due to mishandling in transportation), check
following points before you call your Telex dealer:

i. Are you sure your Telex is on? Check the on-off switch and the volume control.

2. Have you checked your battery? Always carry a spare battery with you.

3. Is there wax in your ear tip? If so, the sound cannot get through. Remove it from the instrument and clean
the tip with Tele-Kleen. Tele-Kleen.

4. Is there wax in your ear canal? Occassionally check your ear for normal accumulation of wax. Visit your doctor periodically for an ear examination.

O estudo histórico nos leva a discussão da necessidade de resgate de temas anteriormente abordados e que talvez ainda sejam necessários se considerarmos o estigma ao deficiente auditivo, ainda presente em nossa sociedade.

O sucesso de materiais impressos está ligado ao correto uso de estratégias de comunicação, que tem a finalidade de melhorar a compreensão dos pacientes e, por conseguinte, auxiliar no sucesso do tratamento. A interface entre o Design e a Fonoaudiologia mostra a importância da busca por soluções gráficas que contribuam com o bem estar do 
paciente, destacando-se a importância de estudos de design voltados a materiais de promoção à saúde.

\section{Referências Bibliográficas}

Artigos em revistas acadêmicas/capítulos de livros

BROOKE, R. E. et al. 2012. Hearing aid Instruction Booklets: Employing Usability Testing to Determine Effectiveness. Am J Audiol, v.21, n.1: 206-214.

BULL, F. C. et al. 2001. Understanding the effects of printed health education materials: Which features lead to which outcomes? J Health Commun,v.6, n.3: 265-279.

CAPOSECCO, A.; HICKSON, L.; MEYER, C. 2014. Hearing aid user guides: Suitability for older adults. Int J Audiol, v. 53, n. S1: S43-S51

DOMICIANO, C.L.C. 2012. Produção Gráfica: Dos primeiros sistemas de reprodução às linguagens digitais. In: Bauru: Canal 6:.62-89 . (Org.). Ensaios em Design. Produção e diversidade.

MUDRY, A.; DODELÉ, L.2000. History of the technological development of air conduction hearing aids.J Laryngol Otol. v.114, n.6: 418-23.

\section{Livros}

AMBROSE, G.; HARRIS, P. 2012. Fundamentos de Design Criativo. 2ª . Edição. Porto Alegre, Bookman.

BANN, D. 2010. Novo Manual de Produção Gráfica. Porto Alegre, Bookman,

LYBARGER, S. F., LYBARGER, E. H. 2000. A historical overview. In Sandlin R. Textbook of Hearing aid amplification. 2 ed. Singular Publishing, San Diego.

LUPTON, E. 2013. Pensar com tipos. 2. ed Cosac Naif. São Paulo.

SAMARA, T. 2011. Guia de Tipografia. Manual prático par ao uso de tipos no design gráfico. Bookman, Porto Alegre.

VILLAS-BOAS, A. 2008. Produção gráfica para designers. 3 ed. 2AB, Rio de Janeiro.

\section{Textos publicados na internet}

CARDOSO, R. O design gráfico e sua história. Artes visuais.

$<$ http://docente.ifrn.edu.br/carlosdias/informatica/programacao-visual/o-design-grafico-e-suahistoria> , 09/12/14

DEAFNESS IN DISGUISE. Bernard Becker Medical Library. Washington University School of Medicine. <http://beckerexhibits.wustl.edu/did/index.htm>, 09/12/14

MUSEU DO APARELHO AUDITIVO. <http://museudoaparelhoauditivo.com.br/acervo-acervodocumental-documentos.php>, 09/12/14

\section{Sobre as autoras}

Camila Medina, mestranda, FOB-USP, Brasil <camila@fob.usp.br>

Deborah Viviane Ferrari, professora associada, FOB-USP, Brasil <deborahferrari@usp.br>

Cassia Leticia Carrara Domiciano, professora doutora, FAAC-UNESP, Brasil

<cassiacarrara@gmail.com> 